\title{
Multilinguales
}

$14 \mid 2020$

Enseignement universitaire et professionnalisation : enjeux, questionnements et défis socio-économiques

\section{Images subliminales et (de)construction du modèle de citoyen camerounais dans les manuels de langues étrangères}

Subliminal images and construction of the Cameroonian citizen model in foreign

language manuals

Vicky Mazoua Megni Tchio

\section{(2) OpenEdition}

\section{Journals}

Édition électronique

URL : https://journals.openedition.org/multilinguales/5729

DOI : $10.4000 /$ multilinguales. 5729

ISSN : 2335-1853

Éditeur

Université Abderrahmane Mira - Bejaia

Référence électronique

Vicky Mazoua Megni Tchio, « Images subliminales et (de)construction du modèle de citoyen camerounais dans les manuels de langues étrangères », Multilinguales [En ligne], 14 | 2020, mis en ligne le 10 décembre 2020, consulté le 02 juillet 2021. URL : http://journals.openedition.org/ multilinguales/5729; DOI : https://doi.org/10.4000/multilinguales.5729

Ce document a été généré automatiquement le 2 juillet 2021. 


\title{
Images subliminales et (de)construction du modèle de citoyen camerounais dans les manuels de langues étrangères
}

\author{
Subliminal images and construction of the Cameroonian citizen model in foreign \\ language manuals
}

Vicky Mazoua Megni Tchio

1 Dans son livre intitulé Didactica Magna, œuvre considérée comme le premier essai important de systématisation de la pédagogie, Comenius insistait, il y a près de 400 ans, sur la nécessité de se servir d'un livre pour enseigner. Pour lui, il s'agissait d'un livre qui combine des lectures adaptées à l'âge des enfants avec des images et des exercices. Comme support visuel, l'image est exploitée en didactique des langues principalement à partir des approches audiovisuelles et s'est étendue considérablement dans le temps avec la prolifération des technologies glottodidactiques. Après la pédagogie audiovisuelle, la conception dynamique de l'apprentissage des langues par l'approche communicative a ramené sur le devant de la scène didactique la problématique de l'image sous toutes ses formes. Le but de notre article est d'analyser la place de l'image dans les manuels de langues étrangères et d'examiner sa perception par les élèves.

2 Selon les usages des différentes époques, de sa nature et de son utilité, le manuel a connu d'importantes fluctuations dans sa définition: Le Petit Robert, par exemple, le définit comme un "ouvrage didactique présentant, sous un format maniable, les notions essentielles d'une science, d'une technique, et spécialement les connaissances exigées par les programmes scolaires ». Choppin, quant à lui, propose une définition des manuels par rapport à leurs fonctions : «ils sont conçus dans l'intention, plus ou moins explicite ou manifeste suivant les époques, de servir de support écrit à l'enseignement d'une discipline au sein d'une institution scolaire» (1992:6). Pour lui, $\mathrm{du}$ fait de sa nature, le manuel est d'abord un produit de consommation, il est aussi le support « du contenu éducatif, le dépositaire de connaissances et de techniques dont la 
société juge l'acquisition nécessaire à la perpétuation de ses valeurs et qu'elle souhaite en conséquence transmettre aux jeunes générations" (Choppin 1992: 6). Porteurs d'une idéologie plus ou moins neutre, les manuels de langue abordent plusieurs domaines (politique, religieux, moral, économique et pédagogique) et pour cela, ils constituent en soi un enjeu des politiques éducatives. Perçus comme tels, les manuels ne sauraient être en contradiction avec les objectifs et la vision de l'Etat en matière d'éducation de ses citoyens. Cependant, il arrive parfois que certaines images présentes dans les manuels pour l'enseignement/apprentissage des langues étrangères dans nos écoles favorisent plutôt, de façon subliminale, un conditionnement affectif négatif visà-vis de certains thèmes essentiels à la formation des jeunes élèves.

3 Le présent article entend interroger la place de l'image dans les manuels pour l'enseignement/apprentissage des langues étrangères à partir d'une double question: qu'apporte de plus, ou de différent, le support image dans une classe de langue ? Et, comment concoure-t-il à l'édification ou plutôt à la déconstruction du modèle d'éducation du citoyen au Cameroun? Pour ce faire, nous tenterons dans un premier temps de définir les fonctions de l'image comme support d'enseignement en classe de langues étrangères pour en préciser les rôles didactiques. Nous analyserons ensuite les effets subliminaux des images les plus représentatives des manuels pour l'enseignement de l'allemand, de l'espagnol et de l'italien dans les établissements scolaires au Cameroun avant de proposer dans un troisième temps une réflexion sur les compétences des auteurs de matériels didactiques à inscrire dans nos programmes officiels.

\section{Images et classe de langues étrangères}

4 Suivant une dichotomie liée au contexte d'apprentissage (hors contexte ${ }^{1}$ dans notre cas), l'enseignement/apprentissage des langues étrangères subit des contraintes liées aux variables environnementales. Ces facteurs contextuels concernent principalement le manque d'infrastructures (salles de classe, instruments technologiques); le volume horaire réservé aux langues étrangères ( $3 \mathrm{~h}$ par semaine) ; les effectifs pléthoriques (qui frôlent parfois 100 élèves par classe); l'indisponibilité ou mieux, l'inexistence d'un laboratoire linguistique ; la quantité, la qualité et la fréquence du matériel linguistique en langues étrangères ; les possibilités d'interactions dans la langue cible. Il est évident que ces caractéristiques impactent de façon considérable les résultats d'un processus didactique dont l'objectif est le développement de la compétence communicationnelle, avec une certaine priorité donnée aux habiletés orales. Dans ces conditions, l'usage de l'image associée à des activités langagières spécifiques semble être le support didactique privilégié pour la réalisation des objectifs communicationnels. De nombreuses recherches ont d'ailleurs démontré l'efficacité du langage iconique pour une mémorisation à long terme (Danesi, 1998). S'agissant du rôle de l'image en classe de langue, Tardy (1975) identifie quatre fonctions principales :

- une fonction psychologique de motivation: l'utilisation des supports visuels contribue à rendre plus captivants et plus dynamiques les cours de langue;

- une fonction d'illustration ou de désignation puisqu'il y a association d'une représentation imagée du terme et de l'objet qu'il désigne ;

- une fonction inductrice puisque l'image est assortie d'une invitation à décrire, à raconter, elle joue ainsi le rôle de déclencheur verbal et apparaît alors comme un matériau didactique 
aidant en particulier à l'expression orale de l'élève. Le caractère non discursif de l'image facilite une multiplicité d'interprétations (idées, discussions) et permet à la créativité de se développer ;

- une fonction de médiateur intersémiotique qui apparait comme une sorte de liaison entre deux systèmes linguistiques, la langue maternelle (L1) et la langue-cible (L2), l'enseignant évitant par le biais de l'image de recourir à la traduction.

En somme, la fonctionnalité de l'image est sujette à la position qu'elle occupe dans l'espace global du manuel, aux couleurs et au rapport qu'elle entretient avec la thématique illustrée.

\section{Corpus et méthodologie de recherche}

L'analyse d'image prend nécessairement en compte le langage verbal, c'est-à-dire le texte et le rapport intrinsèque qu'il entretient avec l'image dans la mesure où dans la plupart des cas, c'est sa verbalisation qui donne un sens à l'image. Roland Barthes (1964) transpose à l'étude de l'image les concepts d'analyse de la sémiologie du discours tels que l'organisation rhétorique, le signifiant et le signifié, la dénotation, la connotation, la polysémie et les fonctions d'ancrage et de relais.

Selon la classification des signes opérée par Peirce, l'image appartient à la catégorie d'icône et est liée à son référent par un rapport de similitude, d'analogie. En effet, « un dessin figuratif, une image de synthèse représentant un arbre ou une maison sont des icônes dans la mesure où ils 'ressemblent' à un arbre ou à une maison » (Peirce cit. in Joly, 1993 : 27). L'image renvoie donc à un sens commun avec la particularité qu'on la perçoit principalement par la vue : c'est une représentation visuelle.

Pour Peirce, une image est donc toujours un ensemble de signes qu'il convient d'interpréter au moyen de jugements perceptuels visuels. La signification globale de l'image, par construction ou par convention, dépend principalement de la perception visuelle de l'observateur.

7 Les aspects sémiotiques de l'image concernent également les codes sociaux et les références culturelles et symboliques. En effet, toute image a été réalisée dans certaines conditions socio-économiques dont elle porte les traces, sa lecture nécessite donc la connaissance des codes propres à une culture mais dépend aussi surtout du lecteur, de son expérience, de sa culture, de sa pratique sociale, de son inconscient, de son imaginaire. Ce qui nous intéresse c'est le fait qu'en tant que signe et objet, l'image ne prend son sens que par l'œil d'un regardant. Entre elle et lui, s'établit une relation particulière. On va donc, dans le cadre de cette analyse, s'interroger sur la façon dont les images contenues dans les manuels de langues étrangères interpellent le regardant camerounais.

Pour cela, nous nous appuierons sur la sémiologie dans son orientation pragmatique, dans le but d'étudier le « vouloir-dire » du locuteur entendu ici comme auteur du choix de l'image et son intentionnalité communicative. La sémio-pragmatique, qui voit en la sémiotique et la pragmatique le cadre de référence pour l'analyse de l'image pédagogique, considère que

les signes iconiques ne possèdent pas les propriétés de l'objet représenté, mais ils reproduisent certaines conditions de la perception commune sur la base des codes perceptifs normaux et par la sélection des stimuli qui - ayant éliminé d'autres stimuli - peuvent me permettre la construction d'une structure perceptive. Cette structure perceptive possède - sur la base des codes de l'expérience acquise - la 
même signification que l'expérience réelle dénotée par le signe iconique (Eco, 1970 :

14).

De ce point de vue, l'interprétation de l'image est soumise à de nombreuses procédures d'inférence et de désambiguation, toutes liées à la situation d'énonciation.

Selon l'approche contextuelle de la sémio-pragmatique de Peirce fondée sur sa théorie des signes et des catégories universelles, l'esprit procède toujours par contextualisation pour trouver les significations des choses. Aucun phénomène ne peut exister en « luimême ». Il est toujours dans un monde contextualisé. Le regardant doit mettre en relation les différents éléments de l'image (symbolique des formes et des couleurs) et les éléments des divers contextes, s'il veut aboutir à des conclusions cohérentes.

Notre étude porte sur seize (16) manuels (Tableau 1) pour l'enseignement de l'italien, de l'espagnol et de l'allemand qui ont été inscrits aux programmes des enseignements secondaires au moins une fois dans la période allant de 2008 à 2018. Cette décennie a connu à notre avis beaucoup d'innovations dans le domaine de la glottodidactique au Cameroun, non seulement avec l'introduction des nouvelles langues étrangères ${ }^{2}$ comme l'italien et le chinois dans le sous-système francophone, mais aussi par une prolifération des textes ou manuels destinés à l'enseignement/apprentissage des différentes langues. Un évènement majeur qui a également marqué cette décennie c'est surement la décision du Conseil National d'Agrément du Manuel Scolaire ${ }^{3}$ en vigueur depuis la rentrée scolaire 2018/2019 qui prévoit l'utilisation d'un seul manuel par matière et par niveau d'étude, force d'une certaine manière à se pencher sur la qualité du manuel scolaire au Cameroun. Notre choix s'est également porté sur les manuels des classes de $4^{\mathrm{e}}, 3^{\mathrm{e}}$ et $2^{\text {nde }}$ parce que nous avons estimé que les jeunes destinataires de ces manuels n'ont pas une grande capacité de discernement et peuvent ainsi constituer une cible facile pour les messages subliminaux. En plus, à ces niveaux-là, l'enseignement/ apprentissage des langues étrangères porte sur les structures linguistiques de base (vocabulaire de base, fonctions communicatives) et les thèmes principaux portent sur la vie quotidienne et la société, les images servant à illustrer ces thèmes peuvent contenir des stimuli qui ne sont ni choisis ni traités consciemment. Dans les classes terminales, les programmes proposent plutôt des thèmes d'histoire, de civilisation et de littérature plus enclin à solliciter des illustrations plus neutres.

Dans une analyse basée sur la lecture des images, il peut s'avérer difficile d'établir une limite étanche entre les différents types d'image à prendre en considération dans un texte. On pourrait ainsi distinguer par exemple les photos des graphes, de la bande dessinée, des cartes géographiques etc. François De-La-Bretèque (1992) propose par exemple de distinguer l'image graphique de l'image photographique, l'image unique de l'image en séquence, l'image fixe de l'image en mouvement. Cette contribution ne prend en compte ni les graphismes ni les images en séquence ou en mouvement, à la faveur des photographies qui ont à la fois une fonction indicielle d'enregistrement du réel et une fonction iconique marquée par l'intentionnalité de son auteur et des images fixes.

Le critère principal qui a orienté le choix des images à analyser reste leur utilité illustrative qui met en jeu les mécanismes de la perception. Pour cela, nous avons recensé dans les manuels pris en examen, principalement des photographies et des dessins en évitant les illustrations récurrentes servant à signaler une activité ou une consigne. 
Tableau 1 : liste des manuels analysés

\begin{tabular}{|c|c|c|c|c|}
\hline Discipline & $\begin{array}{l}\text { Titre du manuel } \\
\text { Auteur(s) }\end{array}$ & $\begin{array}{l}\text { Année de publication, } \\
\text { Editeur }\end{array}$ & $\begin{array}{l}\text { Insertion au } \\
\text { programme }\end{array}$ & $\begin{array}{l}\text { Nombre } \\
\text { d'images } \\
\text { analysées }\end{array}$ \\
\hline \multirow{4}{*}{ Italien } & $\begin{array}{l}\text { 1.Didattica dell'italiano. } \\
\text { Classe di } 4 \text {. Livello } 1^{4} \\
\text { M. H. Bikitik }\end{array}$ & $\begin{array}{ll}2015, & \text { L'Harmattan } \\
\text { Cameroun. } & \end{array}$ & Depuis 2016 & 49 \\
\hline & $\begin{array}{l}\text { 2.Didattica dell'italiano. } \\
\text { Classe di 3e. Livello } 2 \\
\text { M. H. Bikitik }\end{array}$ & $\begin{array}{ll}2015, & \text { L'Harmattan } \\
\text { Cameroun. } & \end{array}$ & Depuis 2016 & 68 \\
\hline & $\begin{array}{l}\text { 3. AfrItalia } 1 \\
\text { G. Kuitche Talé }\end{array}$ & $\begin{array}{l}\text { 2016, } \\
\text { Clé }\end{array}$ & 2017-2018 & 102 \\
\hline & $\begin{array}{l}\text { 4.AfrItalia } 2 \\
\text { G. Kuitche Talé }\end{array}$ & $\begin{array}{l}\text { 2016, } \\
\text { Clé }\end{array}$ & $2017-2018$ & 101 \\
\hline \multirow[t]{7}{*}{ Espagnol } & $\begin{array}{l}\text { 1.Nueva Didactica del } \\
\text { Español } 1 \\
\text { H. Bidoung et autres }\end{array}$ & $\begin{array}{l}\text { 2017, Departamento de } \\
\text { Didactica, O. E. Y DD. EE }\end{array}$ & Depuis 2017 & 63 \\
\hline & $\begin{array}{l}\text { 2.Nueva Didactica del } \\
\text { Español } 2 \\
\text { H. Bidoung et autres }\end{array}$ & $\begin{array}{l}\text { 2017, Departamento de } \\
\text { Didactica, O. E. Y DD. EE }\end{array}$ & Depuis 2017 & 151 \\
\hline & $\begin{array}{l}\text { 3.Nueva Didactica del } \\
\text { Español } 3 \\
\text { H. Bidoung et autres }\end{array}$ & $\begin{array}{l}\text { 2017, Departamento de } \\
\text { Didactica, O. E. Y DD. EE }\end{array}$ & Depuis 2017 & 157 \\
\hline & $\begin{array}{l}\text { 4.Excelencia en Español I } \\
\text { C. Tiako Youadjeu } \\
\text { A. M. Manga, D. Bamela }\end{array}$ & $\begin{array}{l}2015, \\
\text { NMI Education }\end{array}$ & $2015-2017$ & 68 \\
\hline & $\begin{array}{l}\text { 5. Excelencia en Español II, } \\
\text { C. Tiako Youadjeu } \\
\text { A. M. Manga, D. Bamela }\end{array}$ & $\begin{array}{l}\text { 2015, } \\
\text { NMI Education }\end{array}$ & $2015-2017$ & 48 \\
\hline & $\begin{array}{l}\text { 6. Excelencia en Español III } \\
\text { E. S. Nganang Ngoudjo } \\
\text { et autres }\end{array}$ & $\begin{array}{l}\text { 2015, } \\
\text { NMI Education }\end{array}$ & $2015-2017$ & 71 \\
\hline & $\begin{array}{l}\text { 7. Majors en Espagnol } 4^{e} \\
\text { G. T. Anaba } \\
\text { N.O. Makwate Tagne } \\
\text { S. Amina }\end{array}$ & $\begin{array}{l}\text { 2015, } \\
\text { ASVA Education }\end{array}$ & $2015-2017$ & 34 \\
\hline
\end{tabular}




\begin{tabular}{|c|c|c|c|c|}
\hline & $\begin{array}{l}\text { 8. Didactica del Español } 1^{5} \\
\text { A. Agra et al }\end{array}$ & $\begin{array}{l}\text { 2000, Departamento de } \\
\text { Didactica, O. E. Y DD. EE }\end{array}$ & $2000-2015$ & 96 \\
\hline & $\begin{array}{l}\text { 9. Didactica del Español } 2 \\
\text { A. Agra et al }\end{array}$ & $\begin{array}{l}\text { 2000, Departamento de } \\
\text { Didactica, O. E. Y DD. EE }\end{array}$ & $2000-2015$ & 107 \\
\hline \multirow{3}{*}{ Allemand } & $\begin{array}{l}\text { 10. Ihr Und Wir } 3 \\
\text { Diallo Mbaye et all. }\end{array}$ & $\begin{array}{l}1993, \\
\text { Buchverlag } \\
\text { Heinevetter GmbH }\end{array}$ & $1994-2011$ & 41 \\
\hline & $\begin{array}{l}\text { 11. Ihr Und Wir Plus: } \\
\text { textbuch } 1 \\
\text { M. Anoumatacky et } \\
\text { autres }\end{array}$ & $\begin{array}{l}2008, \\
\text { Hueber Verlag }\end{array}$ & Depuis 2008 & 36 \\
\hline & $\begin{array}{l}\text { Ihr Und Wir Plus : textbuch } \\
2 \\
\text { M. Anoumatacky et } \\
\text { autres }\end{array}$ & $\begin{array}{l}2009, \\
\text { Hueber Verlag }\end{array}$ & Depuis 2009 & 32 \\
\hline
\end{tabular}

\section{Le concept d'image et la perception subliminale}

$\mathrm{Du}$ point de vue étymologique, le terme subliminal provient du latin sub limen, qui signifie « sous le seuil », c'est-à-dire placer une perception entre le seuil de l'objectivité et celui de la subjectivité. Le subliminal est très souvent lié aux théories du complot et aux manipulations de masse dans la mesure où il susciterait des représentations mentales pouvant influencer notre comportement habituel.

Pour Jean-Baptiste Légal, « on parle d'exposition subliminale quand une information est présentée de telle sorte qu'elle est traitée par notre système perceptif et cognitif, sans pour autant qu'elle soit consciente, même si l'on focalise son attention pour la détecter » (2012: 24). Le message d'une image est donc jugé subliminal s'il est perçu par nos sens mais n'est pas consciemment détecté.

Contrairement à la perception implicite, qui concerne les images cachées qui sont visibles après une analyse profonde et plus attentive, les informations dans le cas de la perception subliminale sont présentées de telle façon qu'elles ne peuvent pas être traitées consciemment, quel que soit le degré d'attention qu'on y porte. Les images subliminales activent donc de façon non consciente des représentations qui leur sont associées et que l'individu qui y est exposé mémorise lui aussi de façon non consciente. En fonction de la nature des représentations activées et de l'activité dans laquelle la personne est engagée, l'exposition produit ou non un effet sur certaines dimensions de son comportement ou de son jugement. On parle d'un stimulus qui atteint le subconscient mais agit sous le niveau de conscience pour influencer nos actions et nos sensations (Merikle, 2000). Les recherches en neuroscience ont en effet démontré que des informations présentées de façon subliminale produisent des effets sur l'activité du cerveau et sur notre activité motrice (Légal, 2012). 


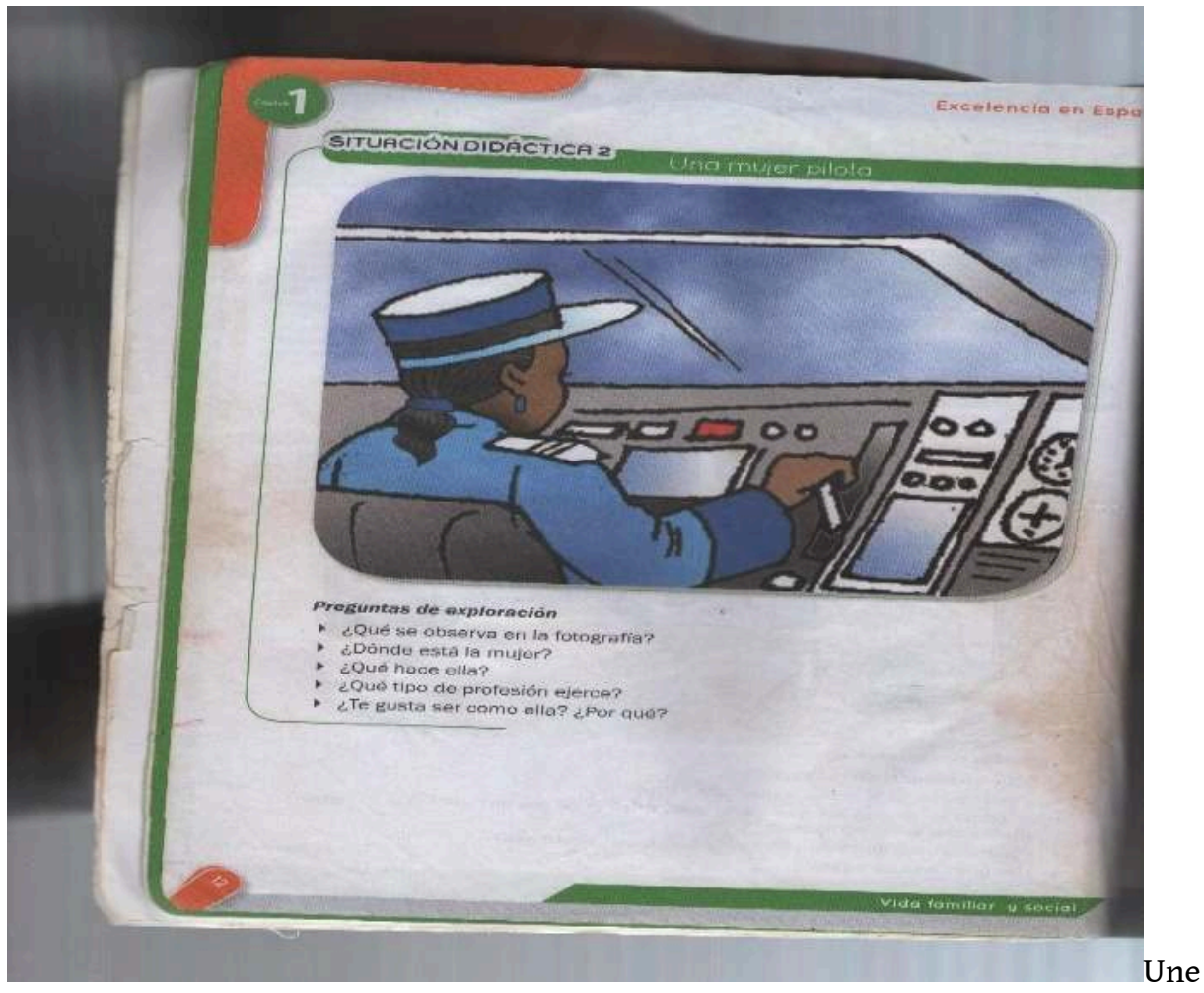

exposition régulière ou répétée à une image subliminale engendrerait un conditionnement affectif capable de modifier notre attitude ou notre jugement vis-àvis d'une personne ou d'un objet: par exemple, si l'image a une teneur émotionnelle (tristesse, joie, peur), elle peut avoir un impact sur notre état émotionnel selon un phénomène de contagion ou d'empathie émotionnelle. On peut ainsi penser qu'une exposition subliminale a un effet d'amplification : notre perception est modifiée, notre attitude est plus tolérante lorsque nous sommes exposés à répétition à un stimulus contenu dans une image. Par exemple, lorsqu'une personne visualise des images liées à la soif (eau, verre, bouteille), ceci accentue très probablement l'envie de boire; idem pour une exposition répétée à des images liées au sexe, à la violence, à l'atteinte à la pudeur. En terrain fertile donc, l'image subliminale contribue à accentuer une attitude, un comportement ou un sentiment.

On peut donc imaginer qu'un adolescent, exposé à une image de manuel qui présente une femme pilote (Image 1) pour laquelle il peut éprouver une totale indifférence au début, sera amené au fil du temps à considérer avec un meilleur sentiment l'idée d'une femme pilotant un Boeing.

\section{Quelques exemples d'images subliminales dans les manuels pour l'enseignement/apprentissage des langues étrangères : une déconstruction du modèle d'éducation du citoyen camerounais}

Les programmes d'enseignement des langues étrangères dans nos écoles présentent dès les premières pages les finalités de cet apprentissage à savoir le développement de la compétence communicative, la connaissance de soi et d'autrui, l'intégration sociopolitique, économique et culturelle $d u$ citoyen camerounais. Ces finalités 
rejoignent les objectifs de la Loi d'orientation de l'éducation de $1998^{6}$ qui dispose en son article 5, alinéa 1 , que l'éducation a pour objectif « la formation de citoyens enracinés dans leur culture, mais ouverts au monde et respectueux de l'intérêt général et du bien commun ». De façon concrète, la sélection du manuel et donc le choix des textes et des images pour le développement de la compétence communicative doit être fait nécessairement à la lumière des missions générales de l'éducation au Cameroun. Les images présentes dans les 16 manuels que nous avons analysés répondent-elles toujours 
à cet objectif? Les messages qu'elles véhiculent sont-ils toujours cohérents avec la vision de l'Etat pour l'éducation de ses citoyens? Citons quelques exemples :

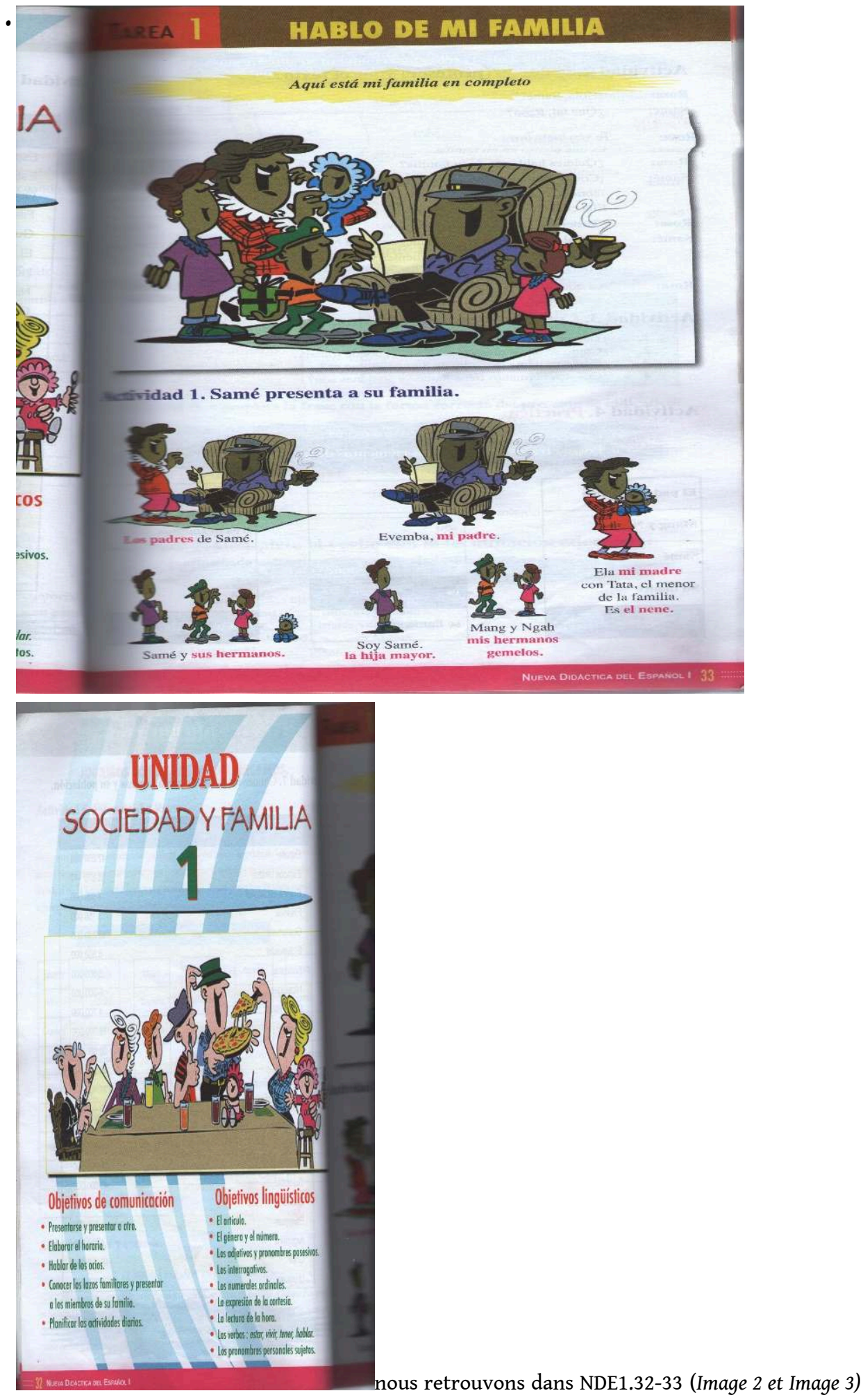

des photos de famille (espagnole et africaine) : A part la couleur de la peau, il n'y a pas de différence au niveau des cheveux alors qu'on sait bien que la texture et la couleur des cheveux afro est bien différente ceux des occidentaux! De façon implicite, une exposition répétée à cette image pourrait justifier le fait que la grande majorité des femmes africaines 


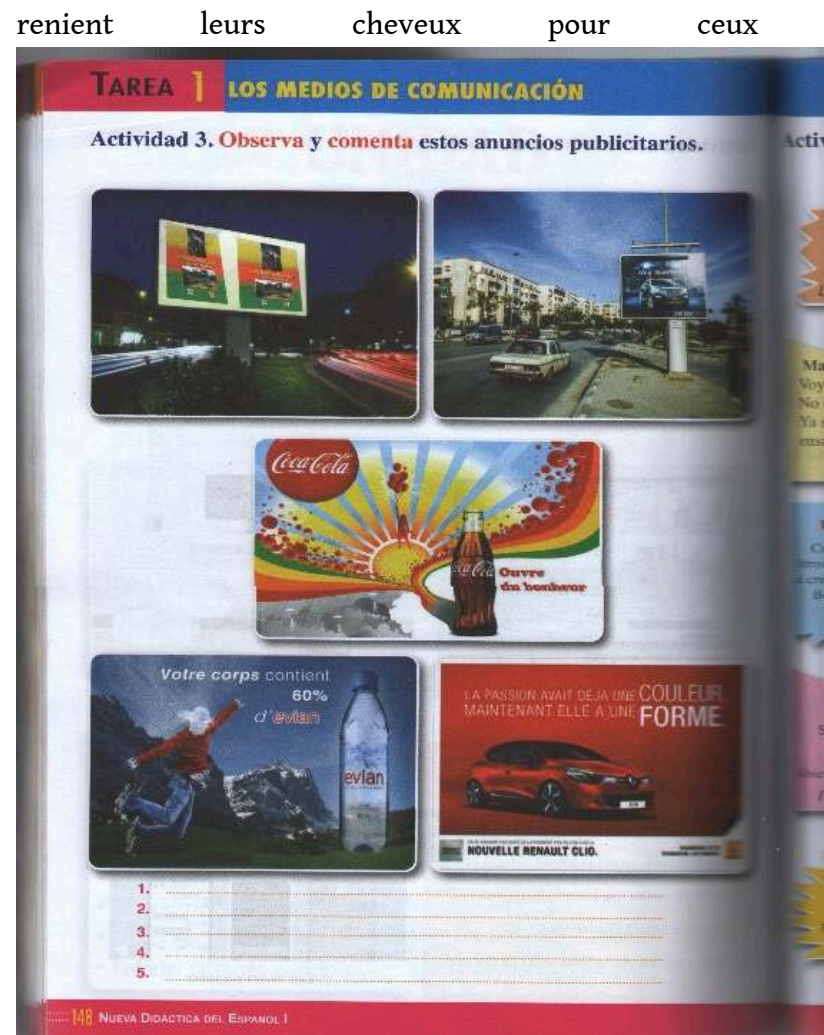

- dans NDE1.148 (Image 4) nous trouvons des images publicitaires des multinationales occidentales : eau minérale EVIAN, une voiture Renault, la boisson gazeuse coca cola qui n'ont aucune connotation culturelle ni avec l'Espagne ni avec le Cameroun. Quelle importance pour les apprenants camerounais? Ces images ne les pousseraient-elles pas à préférer cette eau minérale plutôt qu'une fabrication locale non seulement disponible mais plus avantageuse du point de vue du rapport qualité-prix? 


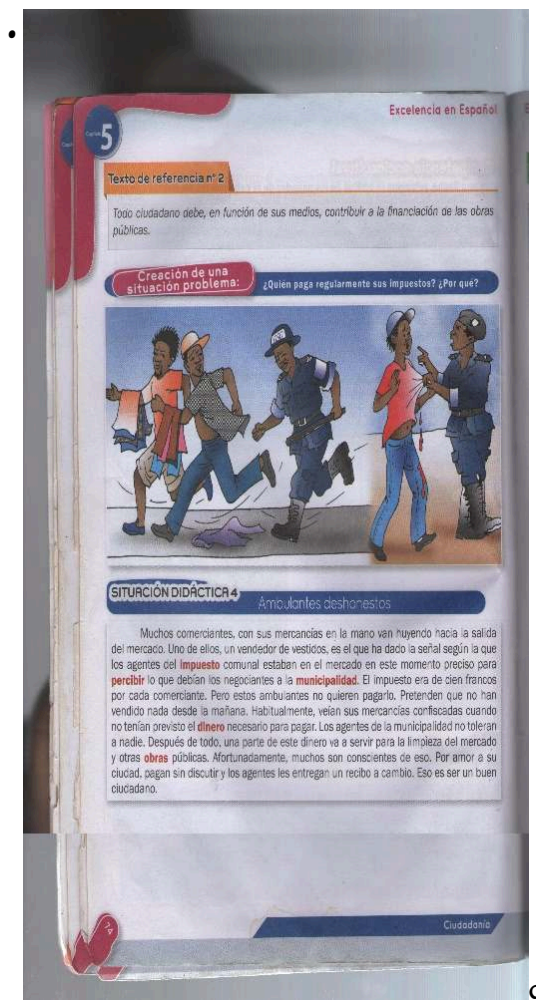

dans EE2.74 (Image 5) nous voyons un policier avec à la main une matraque à la poursuite des vendeurs ambulants et un autre harcelant physiquement un civil qui perd du sang. Une exposition répétée à cette image ne forgeraitelle pas dans l'esprit du jeune citoyen l'idée d'un pouvoir absolu et illimité du corps de la police ? Cette image ne va-t-elle pas à l'encontre de l'article 5 alinéa 5 de la Loi d'orientation qui déclare que "l'éducation a pour objectif l'initiation à la culture et à la pratique de la démocratie, au respect des droits de l'homme et des libertés, de la justice et de la tolérance »? 


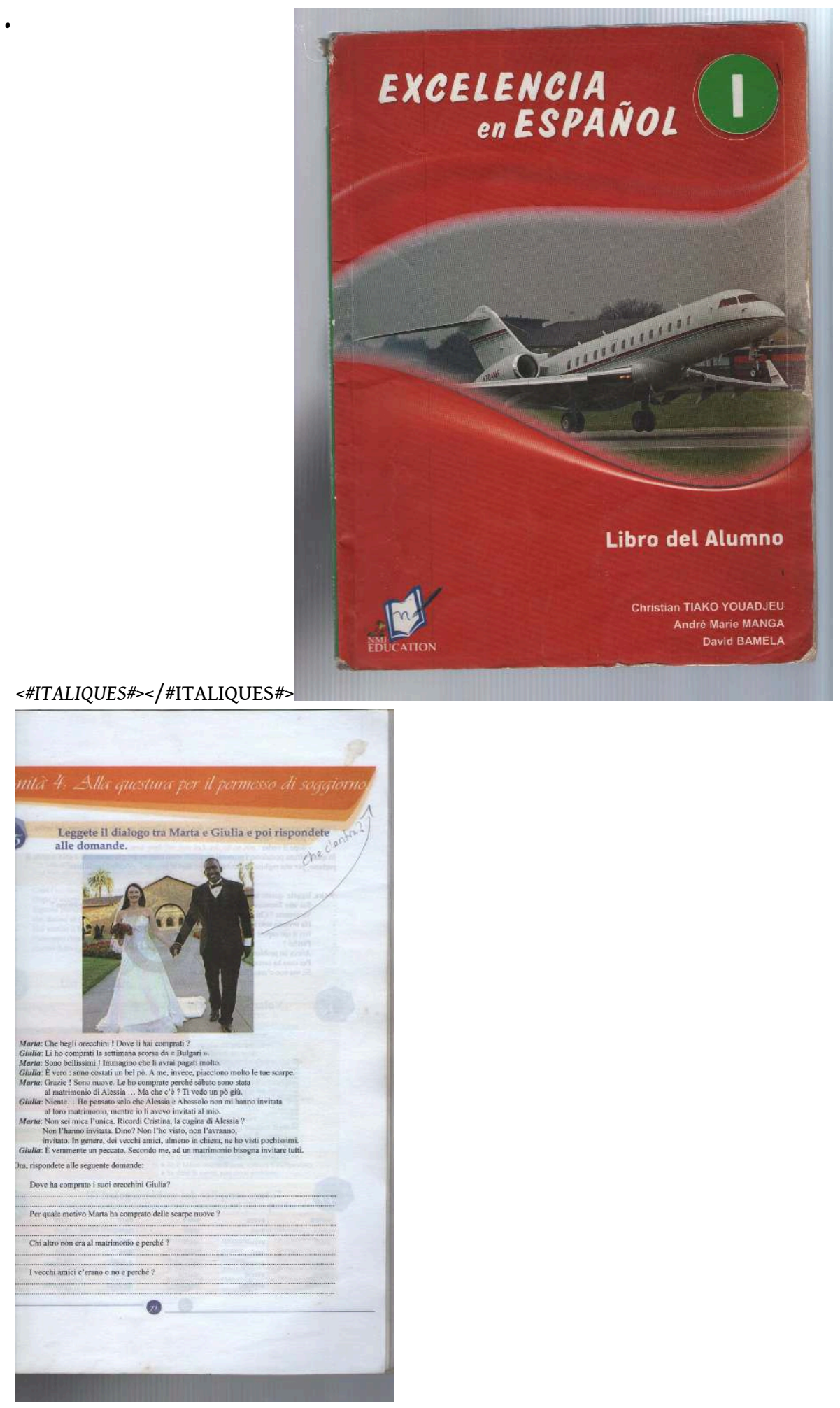




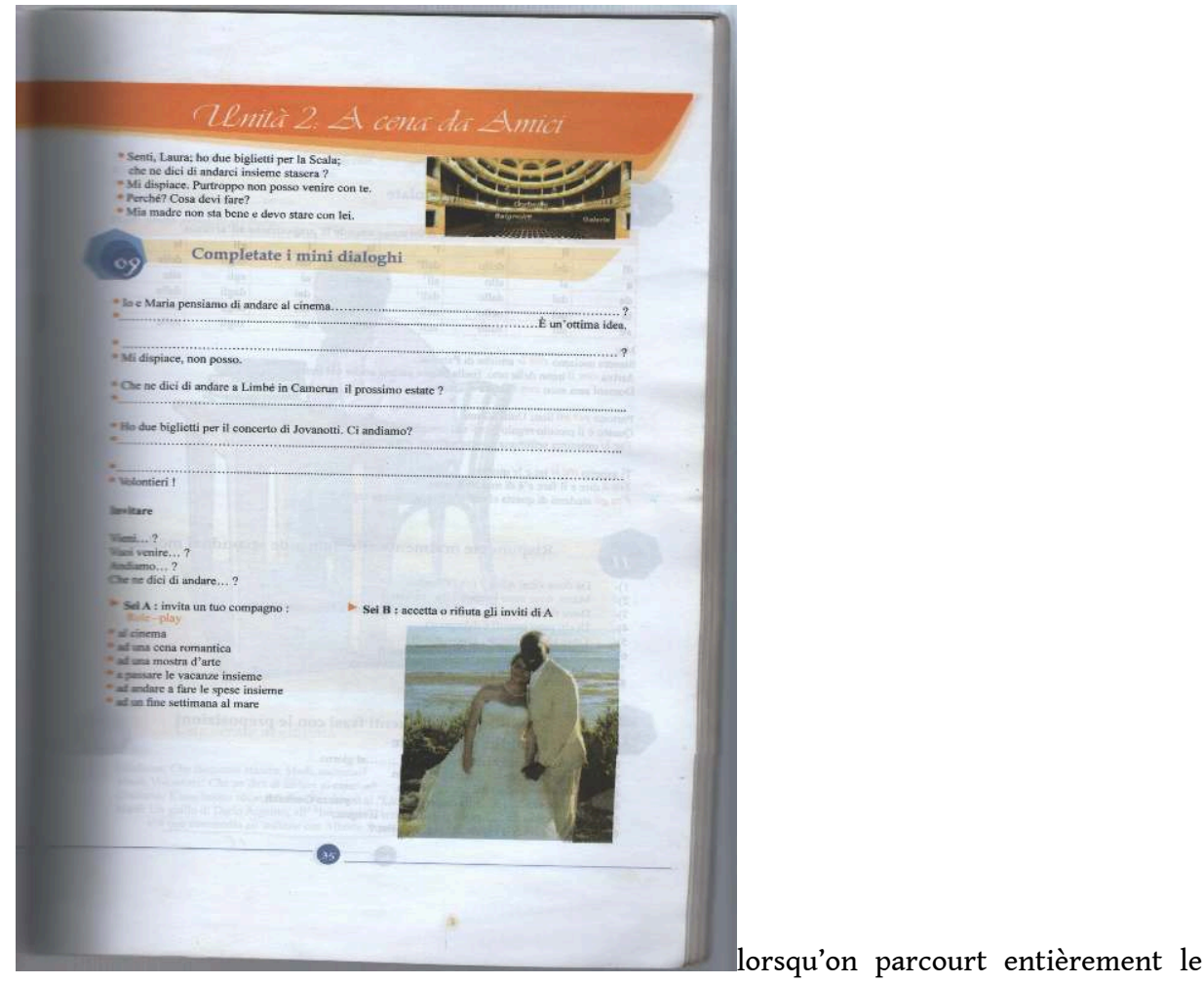
manuel Didattica dell'italiano 2, nous avons deux images (DI2.35, DI2.71) sur le mariage, toutes deux des mariages mixtes (homme noir et femme blanche). Doit-on penser que c'est un idéal à atteindre ? Imaginons donc l'effet sur des adolescents camerounais de 13 ans et 14 ans !

-<\#ITALIQUES\#></\#ITALIQUES\#>

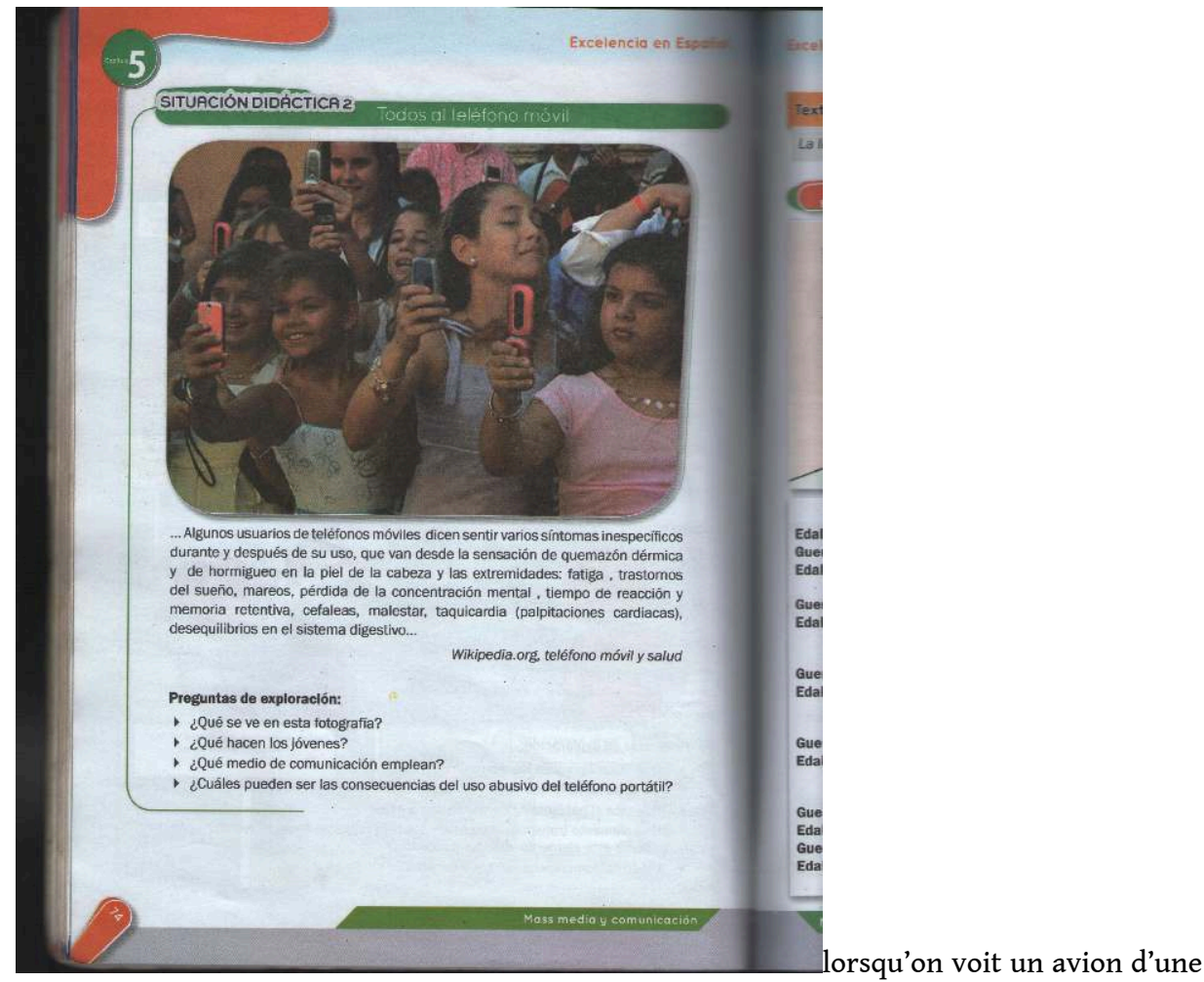

compagnie aérienne étrangère qui décolle en première de couverture du tout premier livre d'espagnol avec lequel l'apprenant entre en contact (Image 8 : EE1.Couverture), ne sommesnous pas en droit de penser que cette image contribue au déracinement des jeunes en les 
incitant à l'émigration? Dans le même manuel, on peut voir à la page 74 (Image 9: EE1.74) des petites filles de moins de 10 ans, souriantes tenant en main des téléphones portables. Loin d'illustrer les conséquences néfastes de l'utilisation du téléphone comme l'indique le texte qui suit, cette image pourrait plutôt emmener l'adolescent camerounais à se demander pourquoi à 14/15 ans il n'a pas lui aussi un téléphone portable !

Des images de ce genre se comptent en dizaine dans la plupart des manuels analysés ; on est en droit de se demander si les auteurs et/ou les illustrateurs de ces manuels sont conscients des messages subliminaux qui transparaissent dans leurs discours iconiques. Le dernier paragraphe interroge les compétences de l'auteur du manuel de langue étrangère.

\section{Du choix conscient de l'image : une réflexion sur la conception du manuel scolaire pour l'enseignement/ apprentissage des langues étrangères au Cameroun}

13 Comme s'il s'agissait d'un livret touristique qui accompagne l'apprenant sur un territoire vaste et inconnu, le manuel de langue doit présenter une sélection utile et représentative des « sites à visiter ». De sa conception jusqu'à sa réalisation, l'auteur du manuel est un guide. Il est le médiateur entre la recherche et l'application, la courroie entre les didacticiens et leurs théories, les politiques linguistiques et étatiques d'une part et d'autre part la classe constituée de l'enseignant et des apprenants. Les manuels qu'il produit doivent prioritairement répondre aux attentes de ces derniers.

Après une analyse des différents manuels en usage dans les écoles et universités africaines pour l'enseignement de l'italien comme langue étrangère, Kuitche observe que la conception du matériel didactique en Afrique subsaharienne est fortement orientée par l'intuition des auteurs. Or, dit-il, les intuitions ne sont utiles que si elles sont informées et confirmées par des analyses et des recherches spécifiques et ponctuelles sur le terrain (Kuitche 2012). En effet, connaitre une langue ne signifie pas savoir enseigner cette langue; de la même façon, être enseignant ne signifie pas automatiquement être capable de planifier, projeter et concevoir l'outil de base d'un enseignement/apprentissage. Un auteur de matériel didactique partage certes beaucoup de compétences avec un enseignant mais il doit démontrer qu'il a une vision globale de la conception de l'ensemble didactique.

Définissant le profil scientifique et professionnel de l'auteur de matériel didactique pour l'EA des LE, Diadori (2011) indique qu'il doit :

- avoir une compétence linguistique à savoir la maitrise de la langue cible ;

- avoir une compétence sociolinguistique et interculturelle (être capable de présenter les aspects de la culture étrangère dans une perspective interculturelle);

- avoir des savoirs en didactique des langues (connaitre les principes de glottodidactique tels que les méthodologies, les méthodes et les techniques qui se sont succédé dans l'histoire de l'enseignement des langues) ;

- avoir des savoirs disciplinaires qui s'inspirent de l'anthropologie culturelle et de la politique linguistique ;

- être capable de projeter des activités qui transforment des savoirs en savoir-faire, en savoir être.

Pour Auger, l'auteur du manuel est un énonciateur qui s'adresse à un co-énonciateur. Il ne connait pas ses co-énonciateurs mais il s'en forge, il s'en fabrique une image 
mentale. C'est à partir de cette image (âge, sexe, idéologie) qu'il définit son intentionnalité communicative (Auger, 2007).

15 Les conditions de production du manuel placent l'auteur dans une position de référence, de modèle. Son discours verbal ou iconique revêt une efficacité symbolique pour l'apprenant qui reçoit le discours de façon presque "aliénante" au sens barthésien du terme. L'auteur du manuel a ainsi le pouvoir de donner sa représentation de la vérité, surtout en ce qui concerne la culture étrangère parfois très distante de l'apprenant du point de vue physique et même psychologique. C'est dans ce cadre qu'Olivier Reboul (1984) définit le discours pédagogique, avec lui celui des supports pédagogiques, comme le plus idéologique de tous les discours. En réalité, s'il est mis au programme des écoles, et donc s'il est choisi pour la formation du citoyen camerounais de demain, le manuel représente un savoir "construit, non problématique, abouti » (Brasquet-Loubeyre, 1994:116) et donc entièrement valide pour le destinataire. Notre analyse a pourtant relevé un bon nombre d'incongruités par rapport aux visées éducatives de notre pays : on va prendre quelques exemples. 


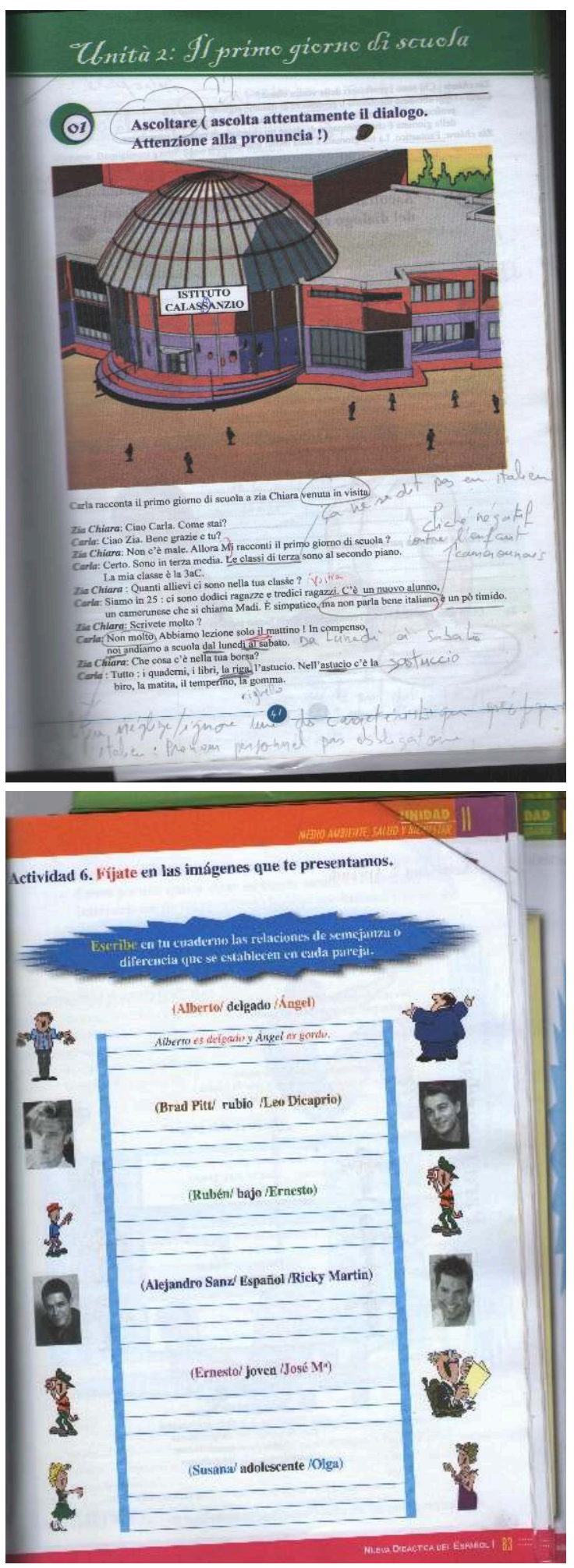




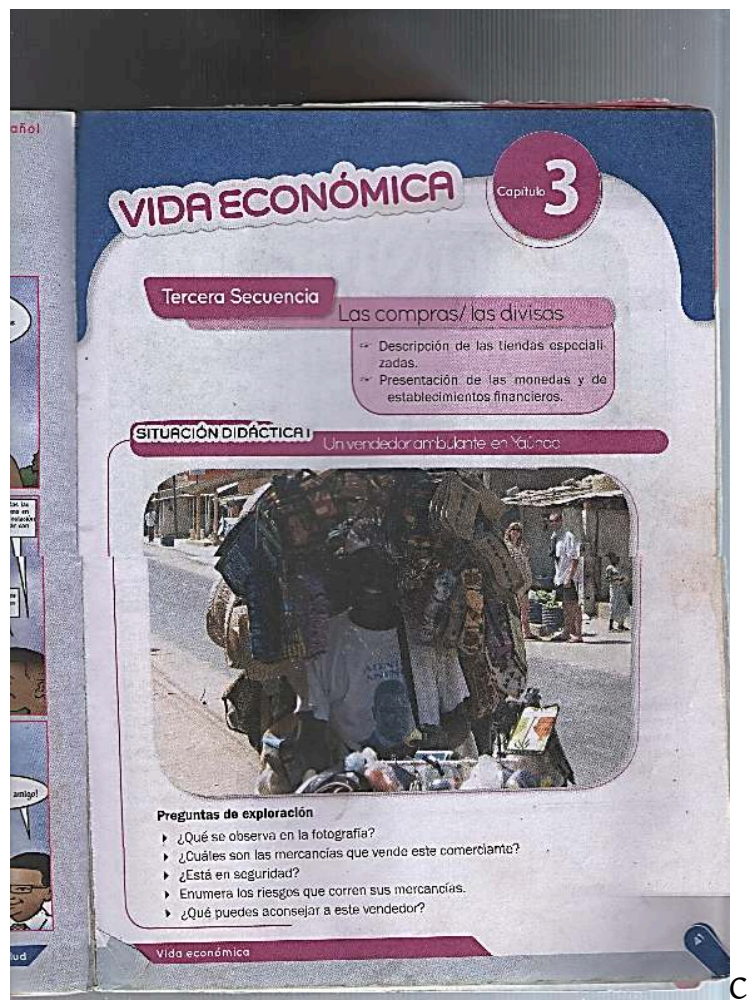
dans le désir peut-être d'illustrer leurs textes, des images qui sont très peu fonctionnelles et à la limite non nécessaires : (Image 10) EE2.41 par exemple présente un vendeur ambulant à Yaoundé (pour ceux qui connaissent la ville de Yaoundé, il est bien difficile de croire qu'il s'agit d'un vendeur ambulant de notre capitale non seulement pour les articles vendus mais aussi pour l'environnement, les constructions.

Dans le souci d'éduquer, peut-être de contextualiser, les images utilisées par d'autres auteurs semblent plutôt contribuer à dé-contextualiser, à déconcerter, à dévier et même dans une certaine mesure à dé-construire :

- dans ND1.83 (Image 11 ci-dessus), pour une activité sur les similitudes et les différences, toutes les images se réfèrent aux personnages publics, acteurs ou artistes musiciens occidentaux, aucun africain et encore moins de camerounais, on est en droit de penser que le manuel ne valorise pas suffisamment la culture locale !

- l'Image 12 (DI1.41) est une image générée ${ }^{7}$ de l'Istituto Calasanzio ${ }^{8}$ de Rome en Italie, pour illustrer le premier jour de classe d'un jeune enfant camerounais de 13 ans en Italie. Cette image contribue sans doute au déracinement 


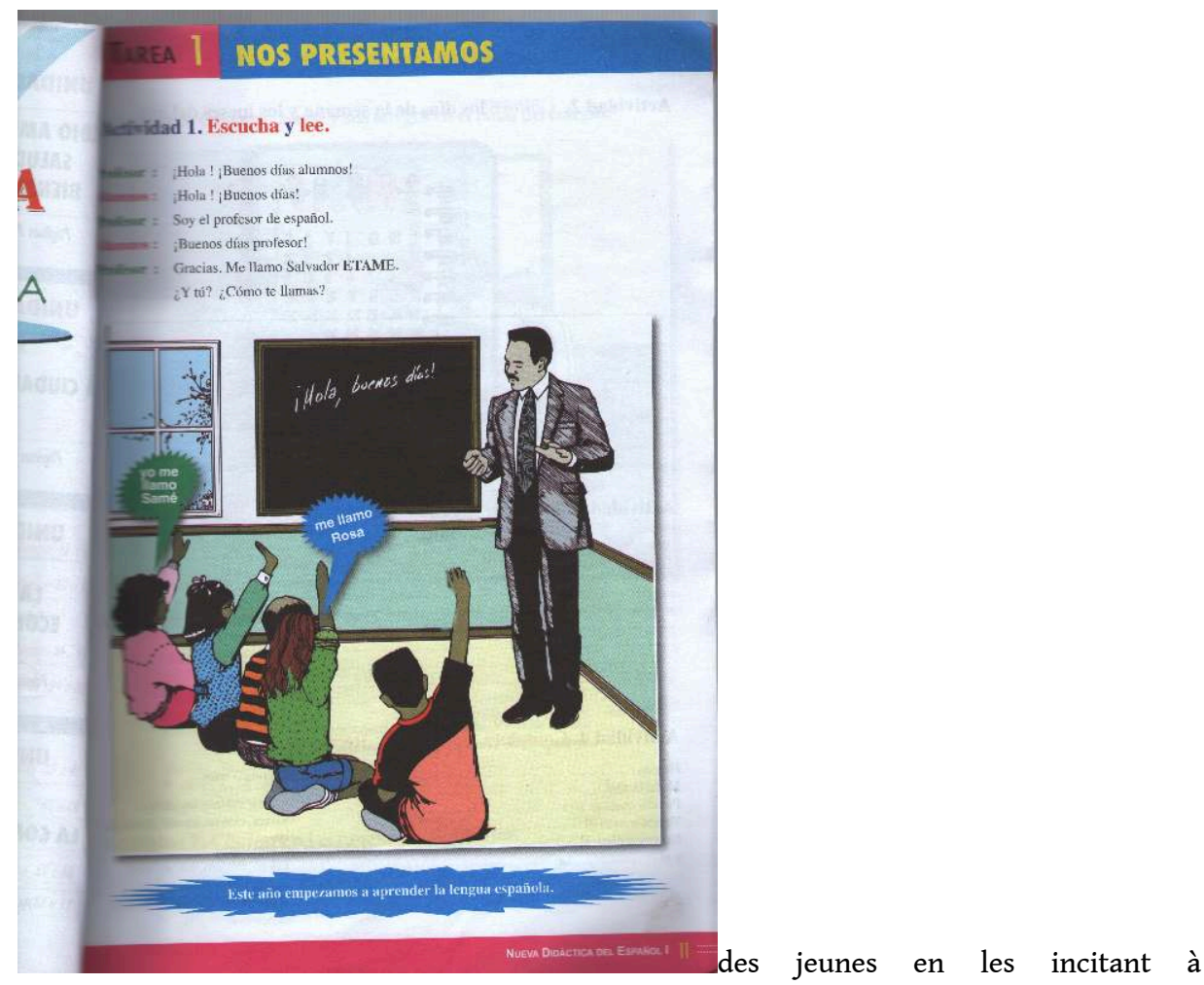

\section{l'émigration ;}

- l'Image $13^{9}$ (DE1.9) présente des enfants assis à même le sol, une situation informelle en contraste évident avec l'enseignant debout en veste cravate (extrême formalité) : un espace classe qui ne se réfère ni aux habitudes de chez nous ni à celles d'ailleurs! Le jeune apprenant de la classe de $4^{\mathrm{e}}$ ne s'identifie pas du tout aux élèves présentés en image et encore moins à l'environnement ;

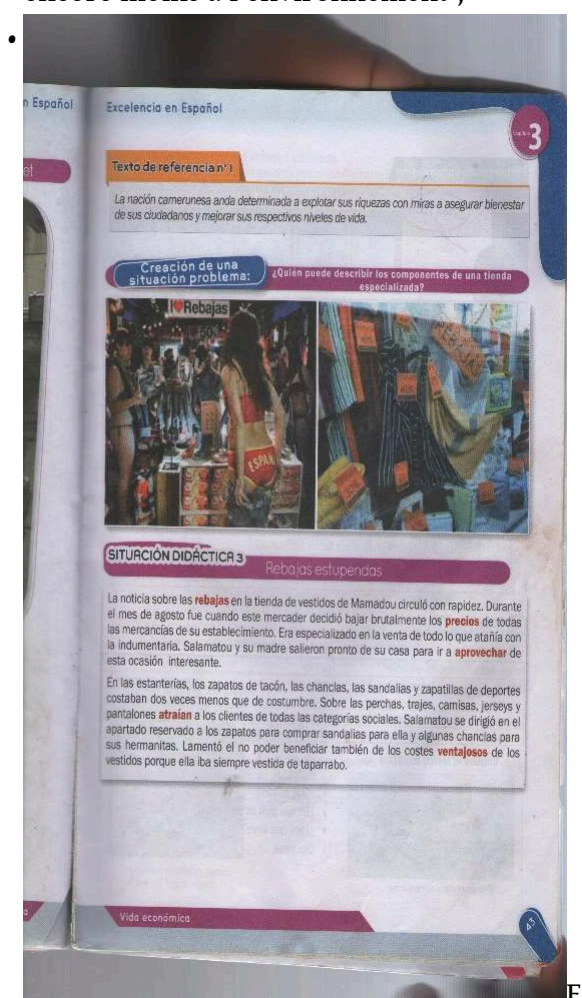

EE2.43 (Image 14) nous présente 2 images pour illustrer les périodes de solde. D'un côté nous avons l'étalage de Mamoudou dont parle le 
texte et de l'autre des femmes occidentales en bikini dans un magasin qui fait des soldes. Il est difficile d'établir la nécessité de cette image pour illustrer les soldes dans une classe d'apprenant ayant entre 13 et 15 ans ! D'autres types de magasins n'auraient-ils pas mieux renvoyé aux soldes?

Cette contribution avait pour objectif de présenter quelques éléments de réflexion sur le pouvoir des messages subliminaux contenus dans les images présentes dans les manuels pour l'enseignement/apprentissage des langues étrangères au Cameroun. Nous avons dans un premier temps rappelé les fonctions de l'image en classe de langue étrangère. Après avoir défini la perception subliminale, nous avons analysé les messages de quelques images répertoriées dans les manuels pris en considération. Notre analyse, encore exploratoire, a porté sur un corpus plutôt réduit qui ne permet pas de généralisation sur les fonctions des images contenues dans ces manuels, mais elle nous a permis de recenser quelques images qui nous ont parues porter un sens connoté en contradiction avec les objectifs de l'éducation déclarés dans les programmes d'enseignement des langues étrangères et dans la Loi d'Orientation de l'Education dans notre pays.

Il ressort de cette analyse que les images subliminales contenues dans les manuels peuvent influencer les choix idéologiques des jeunes apprenants. Le choix des images aussi bien que des contenus - à insérer dans un manuel ne devrait tout simplement pas relever de la contingence mais suivre un parcours de sélection basé sur sa valeur fonctionnelle. Il est clair qu'avec un échantillon plus large, on pourrait arriver à des résultats encore plus significatifs. Il n'en reste pas moins qu'il ne s'agit pas d'une analyse quantitative dans la mesure où chacun des messages subliminaux, en veille dans la mémoire, joue de façon individuelle sur les neurones. Il serait sans doute intéressant de se pencher sur les supports pédagogiques des autres disciplines pour comprendre de façon générale quel pourrait être le degré d'exposition des jeunes apprenants camerounais à ces messages subtils.

\section{BIBLIOGRAPHIE}

AUGER, N., Constructions de l'interculturel dans les manuels de langue, Louvain-la-Neuve, E.M.E, 2007.

AUGER N., «Les manuels : analyser les discours », dans BLANCHET Phillipe, CHARDENET Patrick

(dir(s).), Guide pour la recherche en didactique des langues et des cultures. Approches contextualisées, Paris, Éditions des archives contemporaines, 2011, pp. 313-316.

BARTHES, R., « Éléments de sémiologie » in Communications, 4, Recherches sémiologiques, 1964, pp. 91-135.

BARTHES, R., «Le message photographique », in Communications, 1, 1961, pp. 127-138.

BARTHES, R., « Rhétorique de l'image », Communication, 4, 1964, pp. 43-44. 
BEACCO, J.-C., « Contextualiser les savoirs en didactique des langues et des cultures », dans BLANCHET Phillipe, CHARDENET Patrick (dir(s)) Guide pour la recherche en didactique des langues et des cultures, Paris, Édition des Archives contemporaines, 2011, pp. 31-40.

BLANCHET, P., CHARDENET, P., Guide pour la recherche en didactique des langues et des cultures, Paris, Édition des Archives contemporaines, 2011.

BRASQUET-LOUBEYRE, M., « Marques de didacticité dans des discours de vulgarisation scientifique à la radio ", Discours d'enseignement et discours médiatiques pour une recherche de la didacticité, in Les cahiers de Cedisor, 2, Presses Sorbonne Nouvelle, 1994, pp. 115-137.

CADET, C., CHARLES R., GALUS J-L, La communication par l'image, Paris, Repères pratiques Nathan, 1990.

CHOPPIN, A., Les manuels scolaires : Histoire et actualité, Paris, Hachette, 1992.

DANESI, M., Il cervello in aula. Neurolinguistica e didattica delle lingua, Perugia, Guerra, 1998.

DE-LA-BRETÈQUE, F., « Image, lecture et didactique », Tréma, 2, 1992, Disponible sur http://

journals.openedition.org/trema/2402, consulté le 30 avril 2019.

DIADORI P., «L'autore di materiali didattici di italiano LS e il suo profilo scientifico-professionale », dans DIADORI Pierangela., GENNAI Caterina, SEMPLICI Stefania (dir(s)), Progettazione editoriale per l'italiano L2, Perugia, Guerra, 2011, pp. 22-55.

Dictionnaire Le Petit Robert, 2010.

ECO U., « Sémiologie des messages visuels », in Communications, 15, 1970, pp. 11-51.

ECO, U., Les limites de l'interprétation, Paris, Grasset, 1992.

GERMAIN C., «L'image dans l'apprentissage des langues », in Communication et langages, 29, 1976, pp. 51-68, Disponible sur http://www.persee.fr/doc/colan_0336-1500_1976_num1_4274,

JOLY, M., Introduction à l'analyse de l'image, Paris, Editions Nathan, 1993.

JURADO A.T.C., « L'immagine nei manuali didattici di italiano per stranieri », dans DIADORI Pierangela (dir.), Insegnare italiano a stranieri, Milano-Firenze, Mondadori-Le Monnier, pp. 342-351, 2001.

KOMENSKY, J. A., La grande didactique ou l'art universel de tout enseigner à tous, Paris, Klincksieck, 2002.

LÉGAL J.B., « Le pouvoir des images subliminales », in Cerveau et Psycho, 49, 2012 pp. 24-30, https://www.researchgate.net/publication/279409228_Le_pouvoir_des_images_subliminales, consulté le 21/11/2019.

Loi d'orientation de l'éducation de 1998 http://minedub.cm/uploads/media/ LoisArreteEducation.pdf, consulté le 19/11/2019.

MERIKLE, P.M., « Subliminal perception » in Encyclopedia of psychology, 7, 2000, pp. 497-499.

MULLER, C., « La photographie, un outil pour communiquer en classe de langue », in Images et enseignement/apprentissage des langues : le cas du secteur LANSAD, 31, 1, 2012, pp. 10-27.

PLANE, S., « Le manuel, outil d'enseignement, outil d'apprentissages ", in Plane Sylvie, (dir.), Manuels et enseignement du français, Basse Normandie, CRDP, 1999, pp. 65-81.

TARDY, M., « La fonction sémantique des images », in Etudes de linguistique appliquée, 16. Paris, Klincksieck, 1975, pp. 19-43. 


\section{NOTES}

1. Il s'oppose à l'apprentissage en contexte et caractérise les situations d'apprentissage guidé pour lesquelles la langue cible n'est pas langue de communication quotidienne pour l'apprenant. Ce dernier a dans ces conditions la seule voix de l'enseignant et éventuellement le manuel de référence comme uniques sources de matériel linguistique.

2. En 2011 pour l'italien et en 2012 pour le chinois, l'allemand et l'espagnol bénéficient d'une plus longue tradition d'enseignement au Cameroun.

3. https://www.cnamsmd.com/ dernière consultation le 17/03/20.

4. La collection Didattica dell'italiano est constituée essentiellement d'images générées. La grande majorité de photographies sont presque illisibles et donc très peu fonctionnelles pour un cours de langue étrangère.

5. Le manuel est constitué essentiellement d'images générées. La grande majorité de photographies sont presque illisibles et donc très peu fonctionnelles pour un cours de langue étrangère.

6. La Loi d'orientation définit clairement les objectifs, les méthodes et les contenus du système éducatif camerounais sur la base d'idéologies précises et en tenant compte du modèle d'individu que l'Etat veut obtenir au terme du parcours scolaire. Le texte de ladite Loi est téléchargeable en ligne : http://minedub.cm/uploads/media/LoisArreteEducation.pdf.

7. L'auteur aurait pu faire un choix meilleur du type d'image dans la mesure où on trouve sur internet une myriade d'images de ce collège de la ville de Rome en Italie, téléchargeables gratuitement.

8. Écrit dans le manuel Calassanzio, erreur d'orthographe ou choix conscient de l'auteur, nous pencherons pour la première idée dans la mesure où les toponymes et les monuments présentés dans le manuel sont bien réels.

9. On retrouve exactement la même image dans le manuel Nueva Didattica 1 à la page 11 .

\section{RÉSUMÉS}

Les images des manuels d'enseignement/apprentissage des langues étrangères au Cameroun tendent à mettre en exergue les attributs positifs de la culture de l'autre. Elles exposent parfois les jeunes apprenants à des messages subliminaux capables de modifier certaines dimensions de leur jugement. Considérant d'une part les compétences de l'auteur du manuel et d'autre part la fonction même de l'école, l'article analyse le pouvoir de l'image subliminale dans la formation du citoyen camerounais.

The images inside coursebooks for teaching/learning foreign languages in Cameroon tend to highlight the positives aspects of the target culture. They sometimes expose young learners to subliminal messages that can modify certain aspects of their perception of the world around them. Considering in the one hand the skills of coursebook's authors and, on the other hand, the real function of school, the paper analyses the impact of subliminal images in the training of Cameroonian citizens. 
INDEX

Mots-clés : image, perception subliminale, manuels scolaires, modèle de citoyen, langues étrangères

Keywords : images, subliminal perception, coursebooks, citizen, foreign languages

\section{AUTEUR}

VICKY MAZOUA MEGNI TCHIO

Université de Maroua, Cameroun 